\title{
Myosin $\mathrm{X}$ and its motorless isoform differentially modulate dendritic spine development by regulating trafficking and retention of vasodilator-stimulated phosphoprotein
}

\author{
Wan-Hsin Lin ${ }^{1}$, Joshua T. Hurley ${ }^{1}$, Alexander N. Raines ${ }^{2}$, Richard E. Cheney ${ }^{2}$ and Donna J. Webb ${ }^{1,3, *}$ \\ ${ }^{1}$ Department of Biological Sciences and Vanderbilt Kennedy Center for Research on Human Development, Vanderbilt University, Nashville, \\ Tennessee 37235, USA \\ ${ }^{2}$ Department of Cell Biology and Physiology, University of North Carolina, Chapel Hill, North Carolina 27599, USA \\ ${ }^{3}$ Department of Cancer Biology, Vanderbilt University, Nashville, Tennessee 37235, USA \\ *Author for correspondence (donna.webb @ vanderbilt.edu) \\ Accepted 19 July 2013 \\ Journal of Cell Science 126, 4756-4768 \\ (c) 2013. Published by The Company of Biologists Ltd \\ doi: 10.1242/jcs. 132969
}

\section{Summary}

Myosin X (Myo10) is an unconventional myosin with two known isoforms: full-length (FL)-Myo10 that has motor activity, and a recently identified brain-expressed isoform, headless (Hdl)-Myo10, which lacks most of the motor domain. FL-Myo10 is involved in the regulation of filopodia formation in non-neuronal cells; however, the biological function of Hdl-Myo10 remains largely unknown. Here, we show that FL- and Hdl-Myo10 have important, but distinct, roles in the development of dendritic spines and synapses in hippocampal neurons. FL-Myo10 induces formation of dendritic filopodia and modulates filopodia dynamics by trafficking the actin-binding protein vasodilator-stimulated phosphoprotein (VASP) to the tips of filopodia. By contrast, Hdl-Myo10 acts on dendritic spines to enhance spine and synaptic density as well as spine head expansion by increasing the retention of VASP in spines. Thus, this study demonstrates a novel biological function for Hdl-Myo10 and an important new role for both Myo10 isoforms in the development of dendritic spines and synapses.

Key words: Synapse, Dendritic spine, Myosin, Filopodia, Hippocampal neuron

\section{Introduction}

Dendritic spines are actin-rich protrusions that function as postsynaptic compartments for most excitatory synapses in the brain (Gray, 1959; Harris and Kater, 1994). The development and plasticity of spines underlies cognitive processes, such as learning and memory, and abnormalities in spines contribute to many neurological disorders (Fiala et al., 2002). Spines most likely originate from filopodia-like protrusions, termed dendritic filopodia, which emerge from dendrites and search for presynaptic partners to initiate synapse formation (Ethell and Pasquale, 2005; Ziv and Smith, 1996). After synaptic contact is made, filopodia can then mature into spines. Actin is the main cytoskeletal component in dendritic filopodia and spines, and actin remodeling is crucial for their formation and morphological plasticity (Fischer et al., 1998; Korobova and Svitkina, 2010; Zito et al., 2004). Previous work has focused on the role of the actinbinding proteins that modulate actin dynamics during formation of filopodia and spines (Lin and Webb, 2009); however, much less is known about the function of actin-based motors (myosins) in these processes.

In this context, only a few myosins have been shown to contribute to synaptic processes. Myosin II, the conventional myosin that forms bipolar filaments and crosslinks actin to generate contractility, regulates spine morphology and synaptic function (Hodges et al., 2011; Rex et al., 2010; Rubio et al., 2011;
Ryu et al., 2006). Myosins V and VI, which traffic cargo along actin, are involved in modulating spine function in response to synaptic activity. Specifically, the plus-end-directed motor myosin $\mathrm{V}$ transports vesicular organelles and neurotransmitter receptors into spines to modulate synaptic plasticity (Lisé et al., 2006; Wagner et al., 2011; Wang et al., 2008), whereas the minus-end-directed motor myosin VI regulates the endocytosis of synaptic components at the plasma membrane of spines (Osterweil et al., 2005). However, the contribution of other myosins, such as Myo10, to synaptic function is currently unknown.

Myo10, like other myosins, comprises three regions (Berg et al., 2000): a head domain that binds to actin, hydrolyzes ATP and moves along actin filaments (Kovács et al., 2005; Nagy et al., 2008; Sun et al., 2010); a neck region, consisting of three IQ motifs, that is responsible for binding to calmodulin and calmodulin-like light chains (Homma et al., 2001; Rogers and Strehler, 2001); and a large tail, which includes an $\alpha$-helical region, a motif rich in proline, glutamic acid, serine and threonine (PEST), three pleckstrin homology $(\mathrm{PH})$ domains and a myosin tail homology 4 (MyTH4)-band 4.1/ezrin/radixin/moesin (FERM) supermodule (Hirano et al., 2011; Lu et al., 2011; Wei et al., 2011). While the role of Myo10 in regulating cellular processes is not well understood, it is known to promote filopodia formation and elongation in non-neuronal cells (Bohil et al., 
2006; Pi et al., 2007; Zhang et al., 2004). In neurons, it has been demonstrated to regulate axonal pathfinding, neurite outgrowth and related processes (Ju et al., 2013; Zhu et al., 2007). Recently, a brain-expressed Myo10 isoform, Hdl-Myo10, was identified and shown to have an interesting feature. It lacks the majority of the motor region, but it is otherwise identical to FL-Myo10 (Ju et al., 2013; Raines et al., 2012; Sousa et al., 2006). In contrast to FL-Myo10, the function of Hdl-Myo10 is largely unknown; however, it has been hypothesized to serve as an endogenous dominant-negative of FL-Myo10 because these two proteins share the same domain structure with the exception of the missing motor domain in Hdl-Myo10 (Sousa et al., 2006). Indeed, a recent study showed that Hdl-Myo10 inhibited FLMyo10-mediated axonal outgrowth in cortical neurons (Raines et al., 2012). Nevertheless, it is conceivable that this protein has biological functions, which are independent of FL-Myo10, such as serving as a scaffolding protein, since Hdl-Myo10 has multiple regions that are important for interacting with proteins and lipids (Kerber and Cheney, 2011).

Here, we show that FL- and Hdl-Myo10 are crucial regulators of spine development through differential modulation of the actin-remodeling protein VASP. FL-Myo10 traffics VASP to regulate filopodia formation, whereas Hdl-Myo10 serves as a scaffolding protein that retains VASP in spines to promote spinogenesis and spine head enlargement.

\section{Results}

\section{FL-Myo10 localizes to dendritic filopodia, whereas Hdl-Myo10 localizes to spines}

As scaffolding proteins contribute to the function of spines and synapses, which originate from dendritic filopodia, we hypothesized that Myo10 isoforms, with their multiple domains (Fig. 1A), regulate the development of these structures. To begin to test our hypothesis, we examined the expression of Myo10 in hippocampal neurons (Fig. 1B) during times of spine and synapse formation. Neurons were isolated from embryonic-day 19 (E19) rat embryos and then maintained in culture for 6 (DIV6) and 12 days (DIV12), which are time points where dendritic filopodia and spines are the most prevalent type of protrusions, respectively (Fig. 1C). Both isoforms of Myo10 were expressed in DIV6 and DIV12 neurons, showing that FL- and Hdl-Myo10 are expressed during times of development of dendritic filopodia and spines.

We then assessed the subcellular localization of FL- and HdlMyo10, using live-cell imaging, by coexpressing GFP-tagged Myo10 isoforms along with a fluorescent filler, which allowed visualization of dendritic protrusions (Fig. 1D). Surprisingly, despite having overlapping domains (Fig. 1A), FL- and HdlMyo10 had distinct localizations (Fig. 1D,E). FL-Myo10 was primarily enriched to the tips of dendritic filopodia, whereas HdlMyo10 localized predominantly to dendritic spines (Fig. 1E). Indeed, quantification showed that $77 \%$ of FL-Myo10 puncta localized to filopodia tips and $82 \%$ of Hdl-Myo10 puncta were enriched to spine heads (Fig. 1F). GFP-FL-Myo10 puncta also underwent forward and rearward movement within filopodia, as shown by kymograph analysis (supplementary material Fig. S1A).

To examine endogenous Myo10 localization, we used a Myo10 antibody that recognizes both forms of the protein as isoform-specific antibodies are not available. In DIV6 neurons, endogenous Myo10 puncta were found at the tips and within the shaft of dendritic filopodia, consistent with the localization of
GFP-FL-Myo10 (Fig. 1D,G). Moreover, in more mature neurons with spines, endogenous Myo10 was enriched to spine heads, which mirrored the localization of GFP-Hdl-Myo10 (Fig. 1D,G). Collectively, our results show distinct localizations for FL- and Hdl-Myo10, suggesting they have different functions in spine development.

\section{FL-Myo10 promotes formation of dendritic filopodia}

To investigate the role of FL-Myo10 in spine development, we generated two short hairpin RNAs (shRNAs) and cloned them into pSUPER vector. Because these shRNAs were designed against the head region of FL-Myo10 that is absent in HdlMyo10, they should be specific for the FL isoform. The FLMyo10 shRNAs knocked down expression of endogenous FLMyo10 by $\sim 70 \%$ compared with empty pSUPER vector or a non-targeting (NT) shRNA (Fig. 2A,B). In addition, the FLMyo10 shRNAs did not significantly affect expression of endogenous Hdl-Myo10, confirming their specificity for FLMyo10 (Fig. 2A,B). Neurons were co-transfected with FLMyo10 shRNAs and GFP as a filler at DIV3-DIV4 and imaged three or four days after transfection (DIV6-DIV7). Knockdown of FL-Myo10 had a significant effect on dendritic filopodia (Fig. 2C). The filopodia density was reduced by $\sim 50 \%$ in neurons expressing FL-Myo10 shRNAs compared with neurons expressing empty pSUPER vector or NT shRNA (Fig. 2D). As dendritic filopodia are precursors of dendritic spines (Ethell and Pasquale, 2005), FL-Myo10 could alter spine and synapse formation through its effect on filopodia. This was addressed by evaluating spine and synaptic density in older (DIV11DIV12) neurons after knockdown of FL-Myo10. Dendritic spines were visualized by staining with phalloidin, which binds to Factin (Allison et al., 1998; Fischer et al., 1998), and synapses were observed by immunostaining for the synaptic vesicle protein SV2 (presynaptic marker) and postsynaptic density protein PSD95 (excitatory postsynaptic marker). Knockdown of FLMyo10 by expression of FL-Myo10 shRNAs significantly decreased the density of spines compared with either empty pSUPER vector or NT shRNA (Fig. 2E,F). Moreover, the number of synapses was reduced by $\sim 50 \%$ in neurons transfected with FL-Myo10 shRNAs compared with either empty pSUPER or NT shRNA transfected neurons (Fig. 2E,GI). Taken together, these results point to a crucial function for FLMyo10 in the development of dendritic spines and synapses by means of the regulation of dendritic filopodia.

\section{FL-Myo10 regulates the dynamics of dendritic filopodia}

During spine development, dendritic filopodia are thought to actively search for presynaptic terminals through their dynamic movement (Ethell and Pasquale, 2005; Ziv and Smith, 1996). This raised the intriguing possibility that FL-Myo10 modulates spine development by regulating the motility of filopodia. We began testing this by transfecting neurons with GFP-FL-Myo10 and assessing its effect on filopodia one to two days after transfection (DIV6-DIV7). GFP-FL-Myo10 was expressed at low levels, less than twofold over endogenous Myo10 (supplementary material Fig. S2A,B). Expression of GFP-FLMyo10 increased the density and length of dendritic filopodia by twofold and 1.6-fold, respectively (Fig. 3A,B). When the length of dendritic filopodia was categorized based on GFP-FL-Myo 10 localization, dendritic filopodia with GFP-FL-Myo10 at their tips were twofold longer than those lacking GFP-FL-Myo10 at the 
tips (supplementary material Fig. S1B). Regulation of filopodia length was dependent on the motor region as its deletion negated the FL-Myo10 effect (supplementary material Fig. S3). In addition, as demonstrated by live-cell imaging, dendritic filopodia were considerably more dynamic in GFP-FL-Myo10expressing neurons as compared with GFP-expressing neurons (Fig. 3C; supplementary material Movies 1 and 2). Quantification showed that the percentage of motile filopodia was increased by approximately fivefold in neurons expressing GFP-FL-Myo10 compared with GFP-expressing neurons
(Fig. 3D). Moreover, GFP-FL-Myo10 expression significantly enhanced filopodia dynamics by increasing the total speed of their movement as well as their speed of extension and retraction (Fig. 3E; supplementary material Fig. S1C). When the speed of filopodia movement was categorized by filopodia length, GFPFL-Myo10 filopodia consistently showed a higher speed of movement than GFP controls in each category (supplementary material Fig. S1D), indicating that the increased filopodia movement in GFP-FL-Myo10-expressing neurons was not simply due to longer filopodia. In addition to enhanced linear

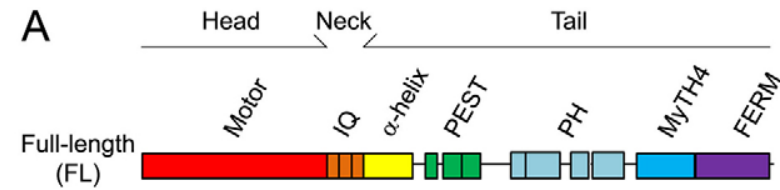

Headless

( $\mathrm{Hdl})$

C

\begin{tabular}{l}
\hline DIV6-8 \\
DII
\end{tabular}

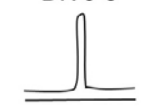

Dendritic filopodia

D

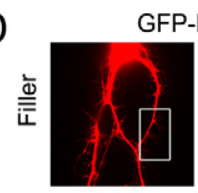

GFP-FL
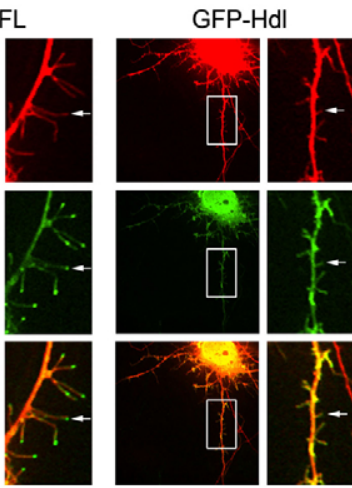

E
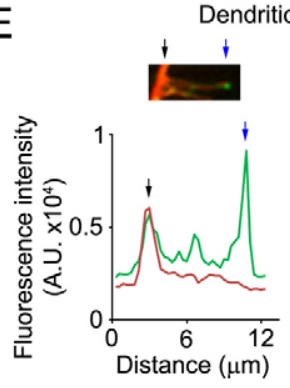

- Filler

- GFP-FL

$\mathrm{F}$
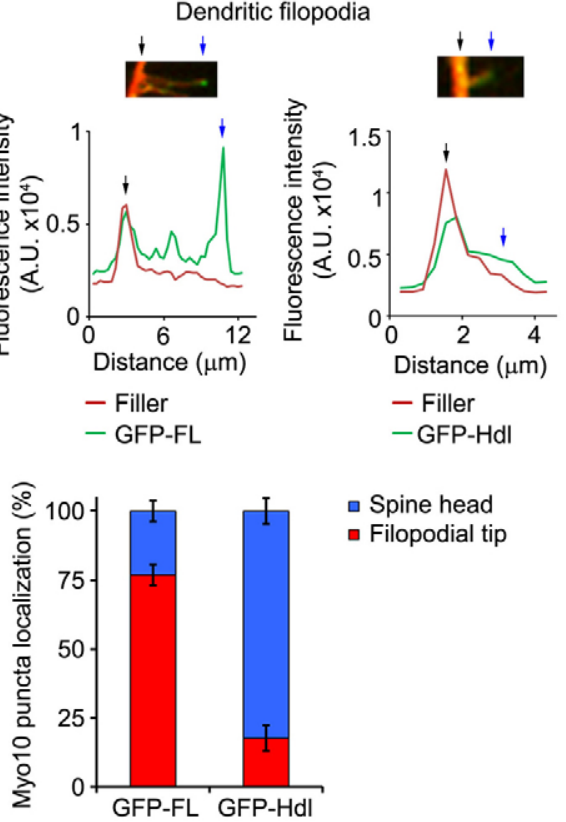

- Spine head • Filopodial tip

G

DIV6
B

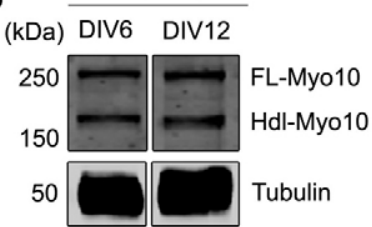

DIV 11-13

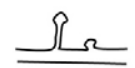

Dendritic spines
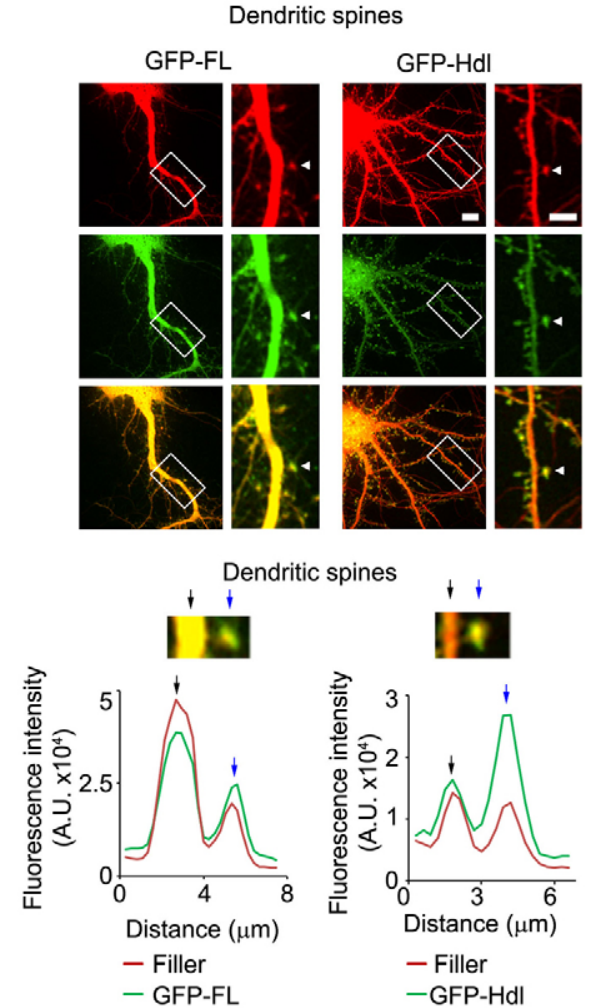

Endogenous Myo10 / F-actin
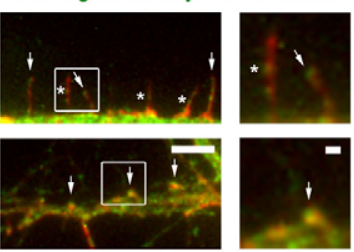

Fig. 1. FL-Myo10 localizes to the tips of dendritic filopodia, whereas Hdl-Myo10 is enriched in spine heads. (A) A schematic illustrating the domains of Myo10 isoforms. (B) Cell lysates from DIV6 and DIV12 cultured hippocampal neurons, which were isolated from E19 rat embryos, were subjected to SDS-PAGE and immunoblotted for Myo10 (upper panels) and $\alpha$-tubulin (lower panels) as a loading control. (C) A schematic denoting the time-period of spine development used in this study. Dendritic filopodia, which are precursors of dendritic spines, are the prevalent type of protrusions at DIV6DIV8, whereas dendritic spines are predominant at DIV11-DIV13. Spines comprise a bulbous head that connects either directly to the dendritic shafts (on right) or by means of a thin neck (on left). (D) Neurons were co-transfected at DIV6 with either GFP-FL-Myo10 or GFP-Hdl-Myo10 and a fluorescent filler, mCerulean. Cells were then subjected to live-cell imaging one and six days after transfection to visualize dendritic filopodia and spines, respectively. mCerulean fluorescent filler is false-colored red. Scale bar: $10 \mu \mathrm{m}$. Higher magnification images of the boxed regions are shown on the right. Scale bar: $5 \mu \mathrm{m}$. Dendritic filopodia (arrows) and spines (arrow heads) are indicated. (E) To illustrate the localization of Myo10 isoforms to the tips of dendritic filopodia and to dendritic spines, linescan analyses were performed. The fluorescence intensities of the fluorescent filler (red) and GFP-Myo10 (green) through the dendrites and along the extended protrusions were graphed as a function of distance. The base (black arrow) and the tip (blue arrow) of dendritic protrusions are indicated. (F) Neurons (DIV5-DIV6) were transfected with GFP-FL-

Myo10 and GFP-Hdl-Myo10, and the subcellular localization of the Myo10 isoforms was examined at DIV8. Quantification of the percentage of GFPFL-Myo10 and GFP-Hdl-Myo10 puncta that localize to the tips of dendritic filopodia and spine heads is shown. Error bars represent the s.e.m. for 26 dendrites from three independent experiments. (G) DIV6 (top panels) and DIV12 (bottom panels) neurons were co-immunostained for endogenous Myo10 (green) and F-actin (red) to visualize dendritic filopodia and spines. Endogenous Myo10 localizes to the tips (arrows, top panels) and shafts (asterisk, top panels) of dendritic filopodia in DIV6 neurons. Myo10 is enriched in spine heads in DIV12 neurons (arrows, bottom panel). Scale bar: $5 \mu \mathrm{m}$. Higher magnification images of the boxed regions are shown (right panels). Scale bar: $1 \mu \mathrm{m}$. 

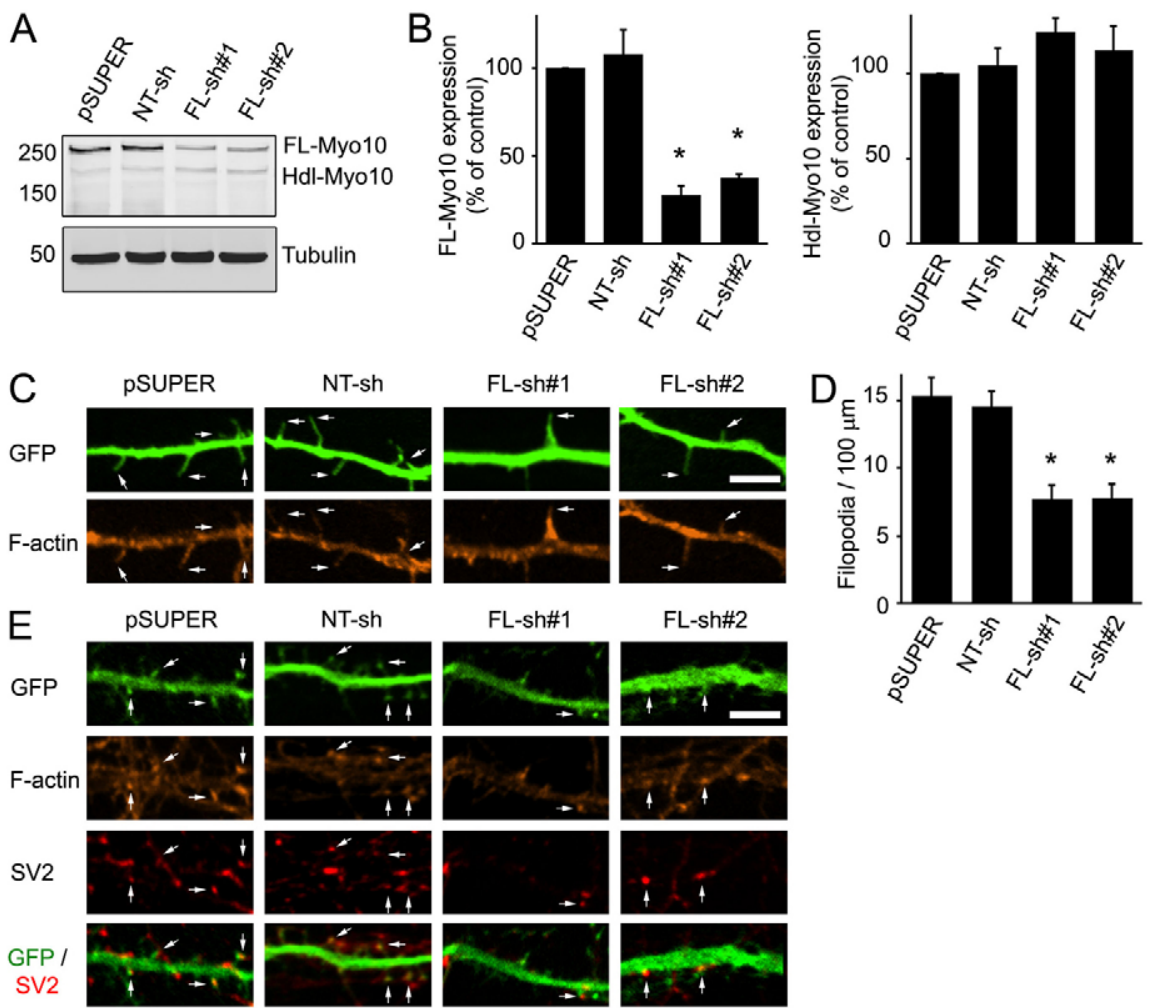

$\mathrm{F}$

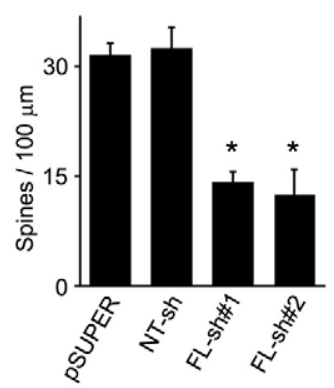

$\mathrm{H}$
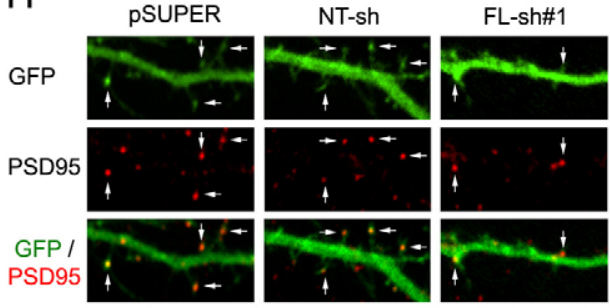
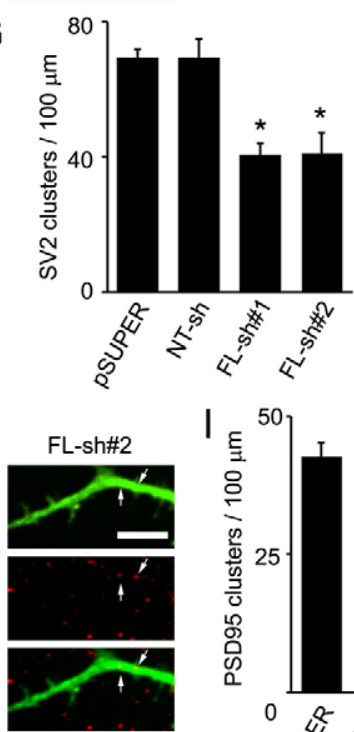

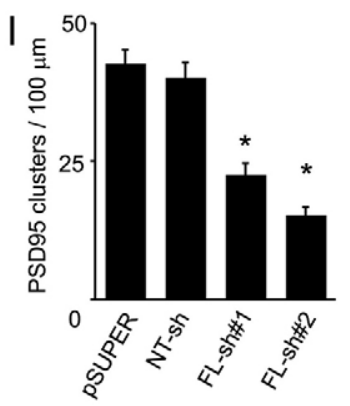

Fig. 2. Knockdown of FL-Myo10 impedes the formation of dendritic filopodia and subsequently spines and synapses. (A) Rat astrocytes (DIV14DIV17) were transfected with empty pSUPER vector, a non-targeting shRNA (NT-sh) or FL-Myo10 shRNAs (FL-sh\#1, FL-sh\#2). Three to five days after transfection, cell lysates were immunoblotted for Myo10 (top panel) and $\alpha$-tubulin (bottom panel) as a loading control. (B) Quantification of endogenous levels of FL-Myo10 (left panel) and Hdl-Myo10 (right panel) from at least three separate experiments is shown. Error bars represent the s.e.m. $\left({ }^{*} P<0.0001\right)$. (C) Neurons (DIV3-DIV4) were cotransfected with GFP and empty pSUPER vector, NT shRNA or FL-Myo10 shRNAs, fixed and stained for F-actin using Alexa Fluor 546 phalloidin at DIV6DIV7. Dendritic filopodia, which are thin protrusions without F-actin enrichment to their tips (arrows), are significantly reduced in neurons transfected with FLMyo10 shRNA. Scale bar: $5 \mu \mathrm{m}$. (D) Quantification of the dendritic filopodia density in neurons transfected with the indicated constructs is shown. Error bars represent the s.e.m. for 40-50 dendrites from three separate experiments $(* P<0.0001)$. (E,H) Neurons (DIV3-DIV4) were co-transfected with GFP and empty pSUPER vector, NT shRNA or FL-Myo10 shRNAs, fixed and stained for F-actin and synaptic markers (SV2, PSD95) at DIV11-DIV12. Spines, which exhibit a bulbous, F-actin-enriched head structure and contact SV2 or contain PSD95 clusters, are indicated (arrows). Scale bar: $5 \mu \mathrm{m}$. (F,G,I) Quantifications of dendritic spine and synaptic density (SV2 and PSD95 clusters) from neurons transfected with the indicated constructs are shown. Error bars represent the s.e.m. for 40-50 dendrites from at least three separate experiments $\left({ }^{*} P<0.0001\right)$. For panels $\mathrm{B}, \mathrm{D}, \mathrm{F}, \mathrm{G}, \mathrm{I}$, asterisks indicate a statistically significant difference compared with pSUPER-transfected cells. extension and retraction, GFP-FL-Myo10 filopodia showed a dramatic increase in their lateral mobility, as shown in Rose plots tracking their movement (Fig. 3F). These results suggest that FLMyo10 is an important modulator of the formation, length and dynamics of dendritic filopodia.

\section{Hdl-Myo10 regulates the formation of dendritic spines and synapses}

As the function of Hdl-Myo10 is largely unknown, and our results indicate that Hdl-Myol0 is enriched to dendritic spines (Fig. 1D-G), we explored the role of this isoform in the formation of spines and synapses by knocking down endogenous expression of Hdl-Myo10. To accomplish this, we generated a shRNA against the $5^{\prime}$-untranslated region (UTR) of Hdl-Myo10 and cloned it into pSUPER vector. Because the 5'UTR is unique to Hdl-Myo10 (Sousa et al., 2006), the shRNA should not affect the expression of FL-Myo10. Indeed, shRNA against Hdl-Myo10 knocked down endogenous expression of this protein by $\sim 40 \%$ without significantly affecting expression of FL-Myo10 (Fig. 4A,B). When neurons (DIV5-DIV6) were cotransfected with Hdl-Myo10 shRNA and GFP, as a filler, and imaged at DIV11-DIV12, a significant effect on spines and synapses was observed. The spine density was decreased by $\sim 50 \%$ in neurons expressing Hdl-Myo10 shRNA compared with 

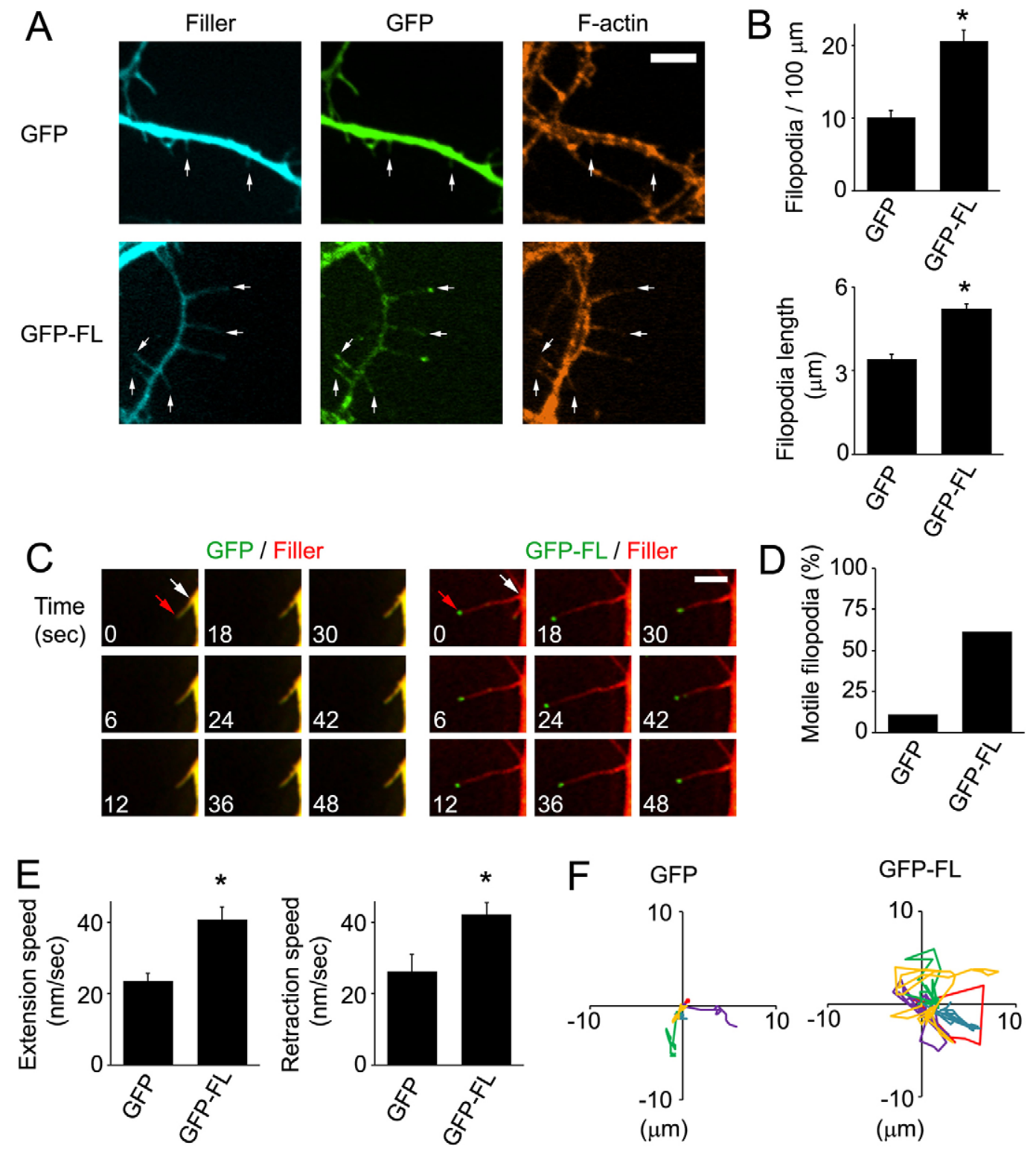

Fig. 3. GFP-FL-Myo10 expression increases the density, length and dynamics of dendritic filopodia. (A) Neurons were co-transfected with either GFP-FLMyo10 (GFP-FL) or GFP and a fluorescent filler, mCerulean, at DIV5, fixed and stained for F-actin at DIV6-DIV7. Dendritic filopodia are indicated (arrows). Scale bar: $5 \mu \mathrm{m}$. (B) Quantifications of the density (top panel) and the length (bottom panel) of dendritic filopodia from neurons transfected with the indicated constructs are shown. Error bars represent the s.e.m. for 40-50 dendrites (top panel) or 100 filopodia (bottom panel) from at least three separate experiments $\left({ }^{*} P<0.0001\right)$. (C-F) Neurons at DIV5 were co-transfected with either GFP-FL-Myo10 or GFP and a fluorescent filler, mCerulean or mCherry, and used for live-cell imaging the next day (DIV6). (C) Images were collected every 3 seconds using a Quorum spinning disk confocal microscope. The montage of images shows the extension and retraction of dendritic filopodia over time. In the first image, the tips (red arrows) and bases (white arrows) of dendritic filopodia are indicated. mCerulean fluorescent filler is false-colored red. Scale bar: $5 \mu \mathrm{m}$. (D) The percentage of motile filopodia was quantified from GFP-FL-Myo10- and GFP-expressing neurons. A total of 68-89 protrusions from at least three separate experiments were analyzed. (E) Quantification of speed of extension and retraction during the recording time is shown. Images were collected every 10 seconds for 10 minutes. Error bars represent the s.e.m. for $68-89$ protrusions from three separate experiments $(* P<0.0001)$. (F) Rose plots tracking the movement of five dendritic filopodia, in different colors, from neurons expressing GFP-FLMyo10 and GFP are shown. GFP-FL-Myo10 expression results in vigorous lateral movement of dendritic filopodia.

neurons expressing empty pSUPER vector or NT shRNA (Fig. 4C,D). Moreover, knockdown of Hdl-Myo10 led to a similar decrease in the number of synapses (Fig. 4C,E-G). Thus, our results suggest that endogenous Hdl-Myo10 regulates the formation of dendritic spines and synapses.

Next, we transfected neurons (DIV5-DIV6) with GFPHdl-Myo10 and assessed its effect on spines and synapses at
DIV11-DIV12. For these experiments, GFP-Hdl-Myo10 expression in neurons was relatively low, only approximately twofold greater than that of endogenous Myo10 (supplementary material Fig. S2C,D). Expression of GFP-Hdl-Myo10 resulted in a 1.5-fold and a 1.3-fold increase in the density of spines (Fig. 5A,B) and synapses (Fig. 5A,C-E), respectively. Deletion of the FERM domain abolished Hdl-Myo10-mediated spine and 

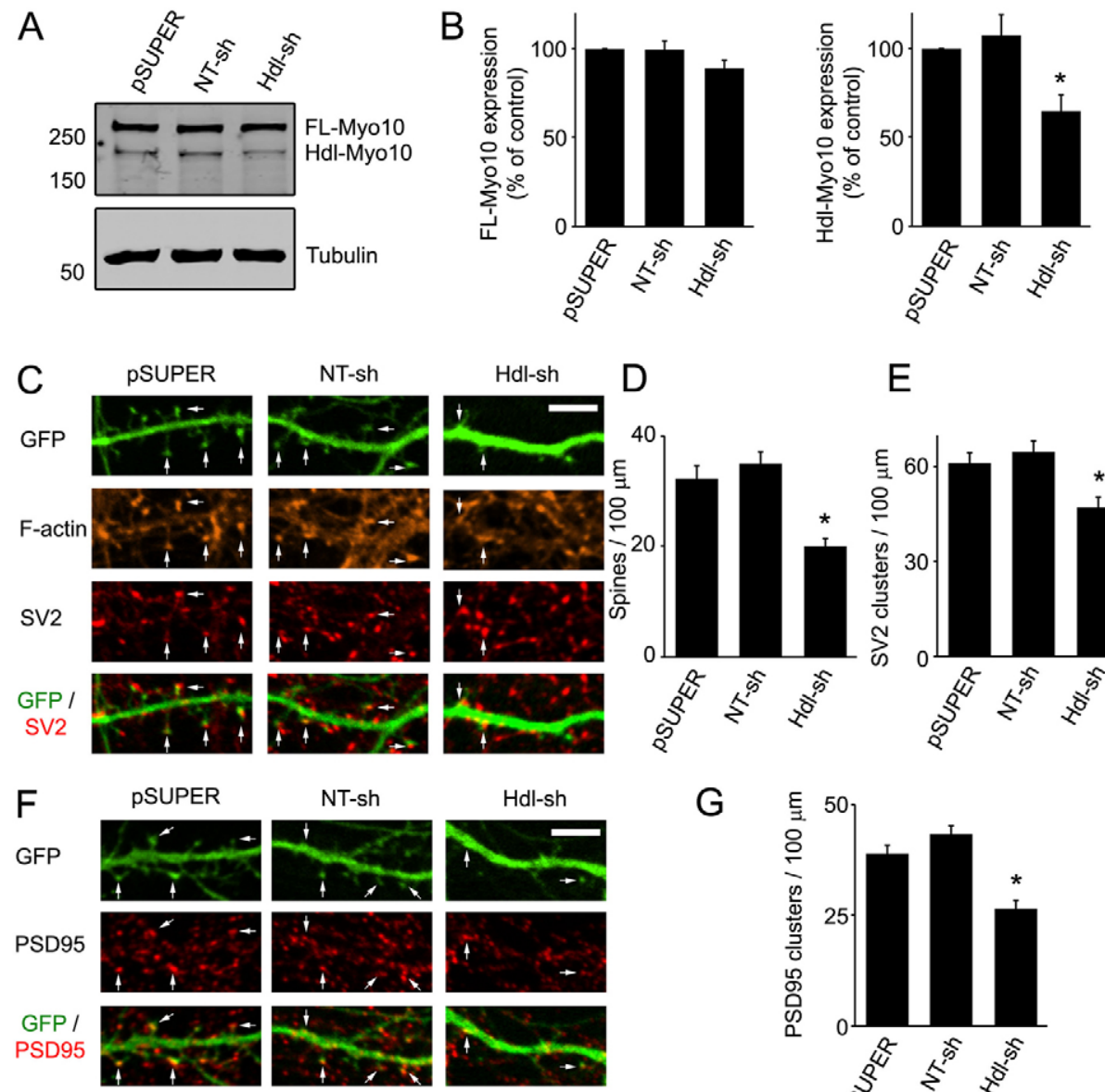

E

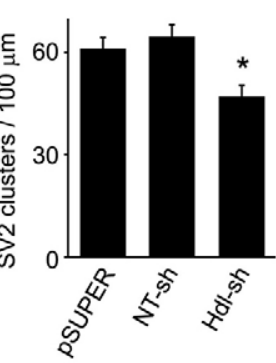

G

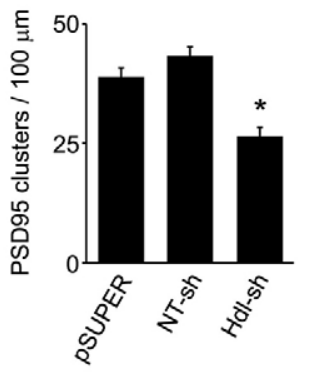

Fig. 4. Knockdown of Hdl-Myo10 impairs dendritic spine and synapse formation. (A) Rat astrocytes (DIV14-DIV17) were transfected with empty pSUPER vector, a non-targeting shRNA (NT-sh) or Hdl-Myo10 shRNA (Hdlsh). Four to five days after transfection, cell lysates were immunoblotted for Myo10 (top panel) and $\alpha$-tubulin (bottom panel) as a loading control. (B) Quantifications of endogenous levels of FL-Myo10 (left panel) and Hdl-Myo10 (right panel) from at least three separate experiments are shown. Error bars represent the s.e.m. $\left({ }^{*} P<0.0015\right)$. $(\mathrm{C}, \mathrm{F})$ Neurons (DIV5DIV6) were co-transfected with GFP and empty pSUPER vector, NT shRNA or Hdl-Myo10 shRNA, fixed and stained for F-actin and synaptic markers (SV2, PSD95) at DIV11DIV12. Spines are indicated (arrows). Scale bar: $5 \mu \mathrm{m}$. (D,E,G) Quantification of dendritic spine and synaptic density (SV2 and PSD95 clusters) from neurons transfected with the indicated constructs is shown. Error bars represent the s.e.m. for $40-50$ dendrites from at least three separate experiments (panels D, G, $* P<0.0001$; panel E, $* P<0.003)$. For panels $\mathrm{B}, \mathrm{D}, \mathrm{E}, \mathrm{G}$, asterisks indicate a statistically significant difference compared with pSUPERtransfected cells. synapse formation, whereas deletion of the PH or MyTH4 domain had no effect (supplementary material Fig. S4). In addition, expression of GFP-Hdl-Myo10 promoted an increase in the size of spine heads, but it did not affect the length of spines (Fig. 5F,G). These data point to an important role for Hdl-Myo10 in regulating the formation of spines and synapses and in modulating synaptic function as expression of this isoform induced the expansion of spine heads. Intriguingly, when GFPHdl-Myo10 was transfected into neurons and filopodia were analyzed at DIV6-DIV8, no differences in the density, length and dynamics of dendritic filopodia were observed between GFPHdlMyo10-expressing and GFP-expressing neurons (Fig. 5H,I; supplementary material Movies 1 and 3). Moreover, higher expression of GFP-Hdl-Myo10 (5-6-fold over endogenous Myo10) did not affect filopodia density (data not shown). In addition, knockdown of endogenous Hdl-Myo10 using HdlMyo10 shRNA had no effect on the density and length of dendritic filopodia (supplementary material Fig. S5A). Therefore, Hdl-Myo10 appears to modulate the development of spines and synapses without affecting the formation or dynamics of filopodia.

\section{VASP interacts with both FL- and Hdl-Myo10}

Our data indicate that FL-Myo10 and Hdl-Myo10 are important modulators of filopodia formation and spine development, respectively, raising the question as to the underlying mechanisms by which they regulate these processes. To date, only a few cargoes have been identified for Myo10 (Almagro et al., 2010; Pi et al., 2007; Tokuo and Ikebe, 2004; Zhang et al., 2004; Zhu et al., 2007). Among the identified cargoes, VASP was an attractive candidate for mediating the Myo10-promoted phenotypes on filopodia and spines because VASP family proteins regulate filopodia formation and spinogenesis by remodeling the underlying actin cytoskeleton (Lin et al., 2010; Lin et al., 2007). Furthermore, VASP is known to co-traffic with FL-Myo10 within filopodia in epithelial cells (Tokuo and Ikebe, 2004). This led us to undertake experiments to determine whether Myo10 regulates spine development through VASP.

We first coexpressed GFP-VASP and mCherry-FL-Myo10 along with a fluorescent filler and examined the localization of VASP and FL-Myo10. GFP-VASP and mCherry-FL-Myo10 were both enriched to the tips of dendritic filopodia (Fig. 6A). Moreover, GFP-VASP and mCherry-FL-Myo10 puncta cotrafficked together at the tips of filopodia (Fig. 6B; supplementary material Movies 4 and 5). In mCherry-FLMyo10-expressing neurons, a twofold increase in VASP localization to filopodia tips was observed compared with mCherry-expressing control neurons (Fig. 6C). Importantly, when the intrafilopodial movement of GFP-VASP was analyzed, expression of mCherry-FL-Myo10 increased the incidence of VASP forward movement by $2.4 \pm 1.5$-fold ( $n=20-32$ dendritic filopodia from three separate experiments). Thus, FL-Myo10 enhances the movement and the localization of VASP to the tips of filopodia. 
A
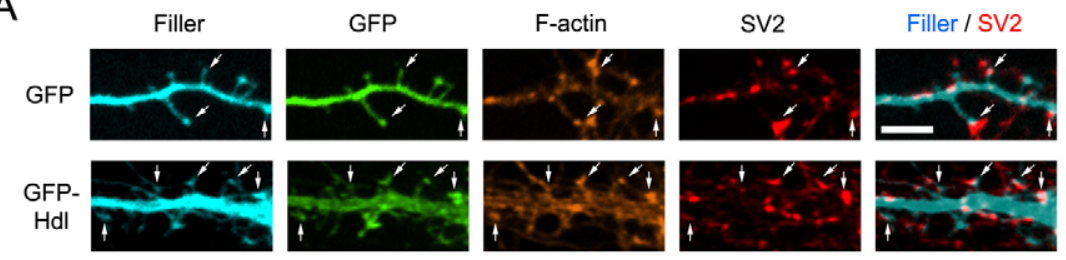

B

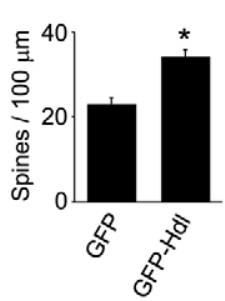

D

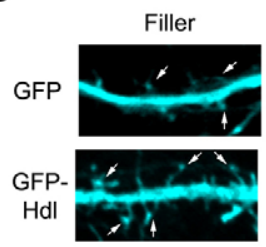

GFP

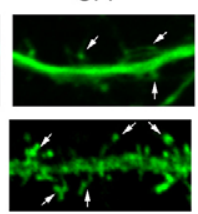

F

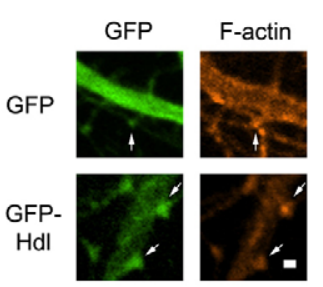

$\mathrm{G}$
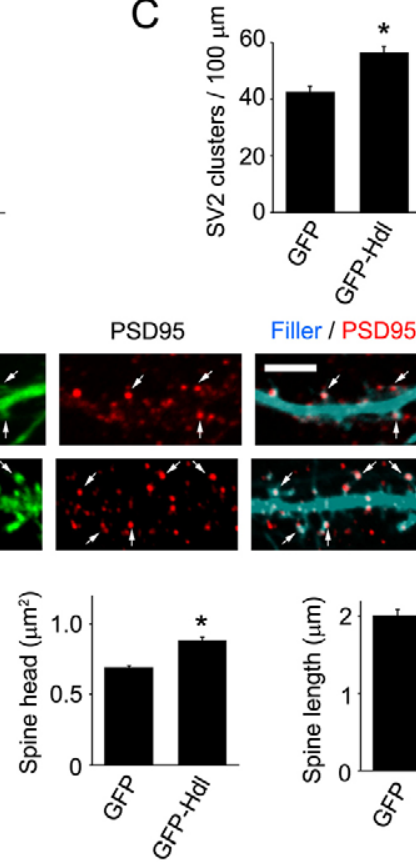

$\mathrm{H}$

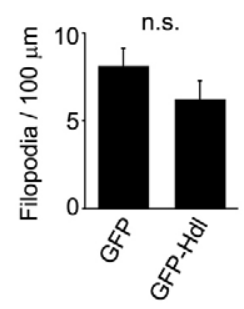

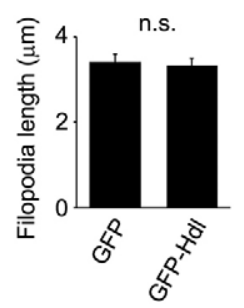

1

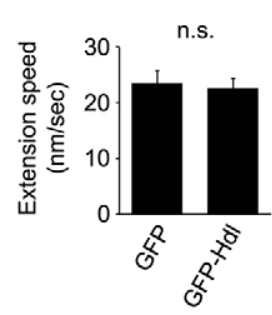

E

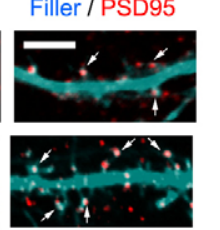

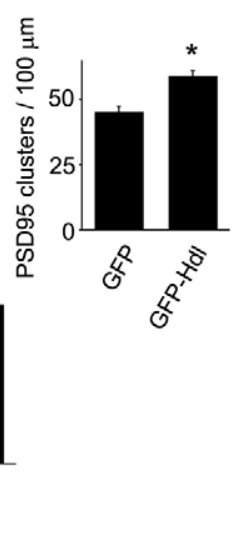
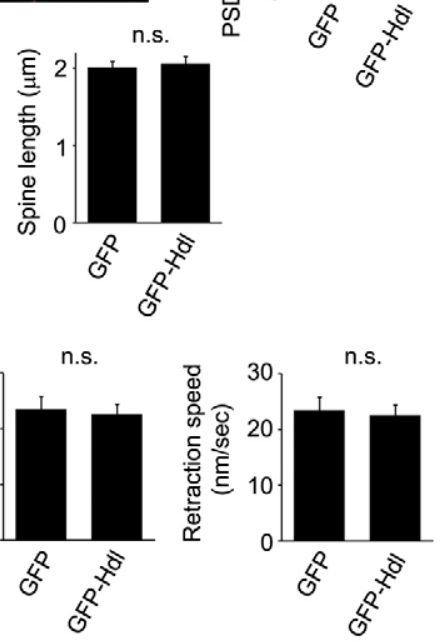

Fig. 5. Expression of GFP-Hdl-Myo10 enhances dendritic spine and synaptic density and increases spine head size. (A-E) Neurons (DIV5-DIV6) were co-transfected with either GFP-Hdl-Myo10 (GFP$\mathrm{Hdl}$ ) or GFP and a fluorescent filler, mCerulean, fixed and stained for F-actin and synaptic markers (SV2, PSD95) at DIV11-DIV12. (A,D) Spine heads have an enrichment of F-actin and colocalize with synaptic proteins (arrows). Scale bar: $5 \mu \mathrm{m}$.

(B,C,E) Quantifications of the dendritic spine and synaptic density (SV2 and PSD95 clusters) from neurons transfected with GFP-Hdl-Myo10 or GFP are shown. Error bars represent the s.e.m. for 43-45 dendrites from three separate experiments $\left({ }^{*} P<0.0001\right)$. (F) Neurons (DIV5-DIV6) were transfected with GFP-Hdl-Myo10 or GFP, fixed and stained for F-actin at DIV11-DIV12. Scale bar: $1 \mu \mathrm{m}$. (G) Quantifications of spine head size and spine length for neurons expressing GFP-Hdl-Myo10 or GFP are shown. Error bars represent the s.e.m. for 200 spines from three separate experiments $(* P<0.0001)$.

(H) Neurons at DIV6-DIV7 were co-transfected with a fluorescent filler, mCerulean, and either GFP or GFP-Hdl-Myo10, and dendritic filopodia were analyzed at DIV7-DIV8. Quantifications of the density (left panel) and length (right panel) of dendritic filopodia from neurons expressing GFP or GFP-Hdl-Myo10 (GFP-Hdl) are shown. Error bars represent the s.e.m. for 33-39 dendrites (left panel) and 102-104 dendritic filopodia from three separate experiments. (I) Neurons at DIV5 were co-transfected with a fluorescent filler, mCherry, and either GFP or GFP-Hdl-Myo10 and subjected to live-cell imaging the next day (DIV6). Images were collected every 10 seconds for 10 minutes. Quantifications of the speed of extension and retraction of dendritic filopodia from neurons expressing GFP or GFP-HdlMyo10 are shown. A total of 63-89 protrusions from at least three separate experiments were analyzed. Error bars represent the s.e.m. for 63-89 protrusions from three separate experiments. For panels G-I, n.s. denotes the absence of a statistically significant difference.
Because Hdl-Myo10 and FL-Myo10 share the same domain architecture except for the motor region, it seemed plausible that Hdl-Myo10 also associates with VASP. Indeed, when GFPVASP and mCherry-Hdl-Myo10 were coexpressed in neurons, they colocalized together in the heads of dendritic spines (Fig. 6D). Knockdown of Hdl-Myo10 using shRNA against Hdl-Myo10 decreased the localization of VASP to spines (supplementary material Fig. S5C,D); however, mCherry-HdlMyo10 expression did not significantly affect VASP localization to filopodia tips (supplementary material Fig. S5B). We next explored the interaction of VASP with the Myo10 isoforms using a biochemical approach. mCherry-VASP was coexpressed with the GFP-Myo10 isoforms or GFP as a control in human embryonic kidney 293T (HEK-293T) cells, and GFP-Myo10 complexes were subsequently immunoprecipitated from cell lysates using an antibody against GFP. As both VASP and FLMyo10 bind to actin, cells were treated with cytochalasin D, which depolymerizes actin filaments, to minimize the possibility that precipitated actin could serve as a link between VASP and
Myo10. VASP was co-immunoprecipitated with both isoforms of Myo10 (Fig. 6E). Surprisingly, a 3.5-fold increase in the amount of VASP was precipitated with Hdl-Myo10 in comparison with FLMyo10 (Fig. 6F). A greater association of VASP with Hdl-Myo10 could result from structural differences between FL- and HdlMyo10. A recent study showed that FL-Myo10 naturally exists in an auto-inhibitory conformation with its PH and FERM domains intramolecularly bound to the motor region (Umeki et al., 2011). Since the tail region, including the $\mathrm{PH}, \mathrm{MyTH} 4$ and FERM domains, of FL-Myo10 binds to VASP (Tokuo and Ikebe, 2004), the auto-inhibitory conformation might alter this interaction. Hence, Hdl-Myo10, which lacks the motor region, might adopt a different conformation that is more conducive to VASP interaction.

\section{Myo10 mediates spine development through VASP}

Because our results suggest that FL-Myo10 traffics VASP to the tips of filopodia, and actin polymerization at this site drives filopodia formation (Mallavarapu and Mitchison, 1999), FL-Myo10 might regulate dendritic filopodia formation through 
A
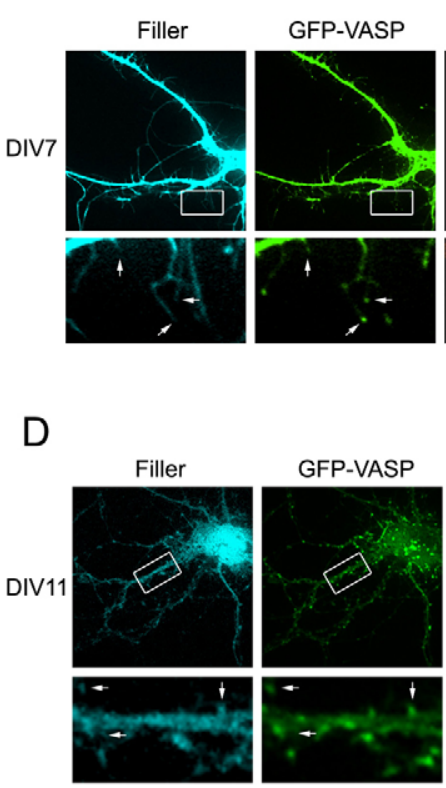

E
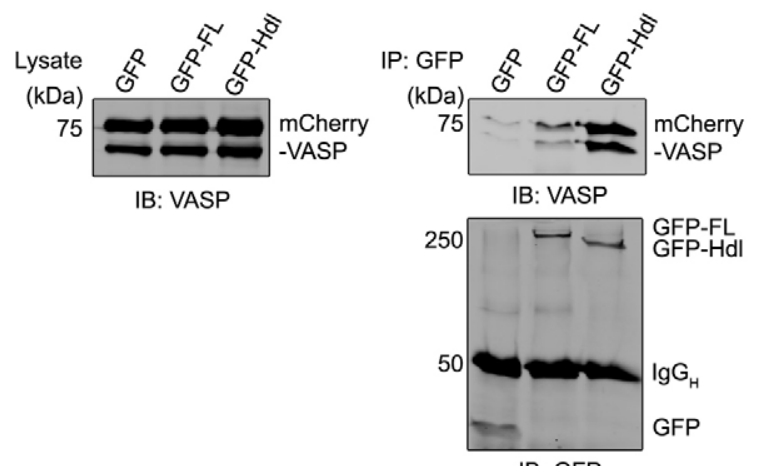

B

Time GFP- mCherry- VASPI

(sec)
0
10
20

30

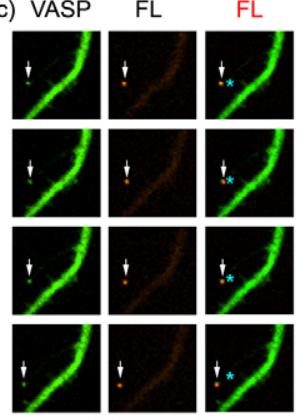

40

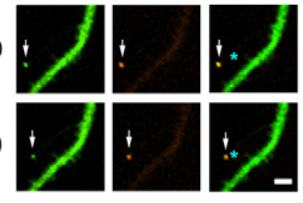

C
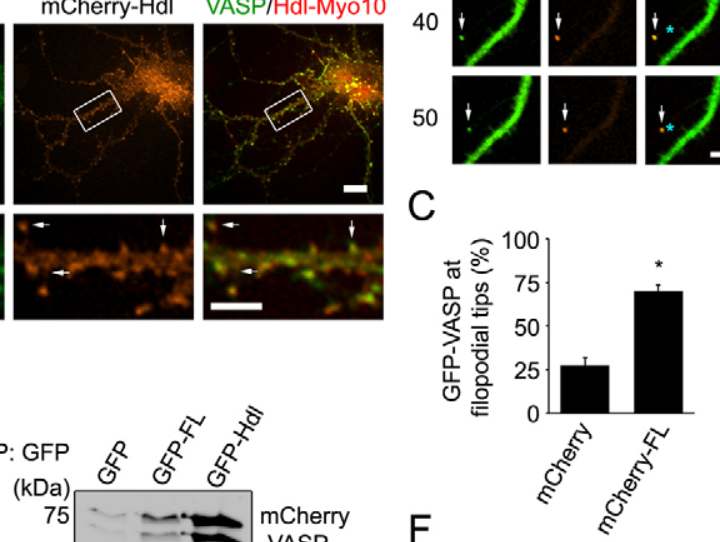

$\mathrm{F}$

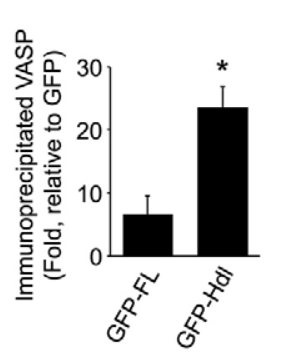

Fig. 6. VASP colocalizes with both FL-Myo10 and Hdl-Myo10. (A) Neurons were co-transfected with GFP-VASP, mCherry-FL-Myo10 (mCherry-FL) and a fluorescent filler, mCerulean, at DIV6 and then subjected to live-cell imaging at DIV7 (upper panels). Scale bar: $10 \mu \mathrm{m}$. Higher-magnification images of the boxed regions are shown (lower panels). Scale bar: $5 \mu \mathrm{m}$. GFP-VASP and mCherry-FL-Myo10 puncta at the tips of dendritic filopodia are indicated (arrows). (B) A dendritic filopodium from a neuron transfected with GFP-VASP and mCherry-FL-Myo10 is shown. The mosaic of images shows a GFP-VASP punctum cotrafficking with mCherry-FL-Myo10 (arrows). Asterisks denote the original position of VASP or FL-Myo10 puncta. Scale bar: $1 \mu \mathrm{m}$. (C) Neurons were cotransfected with GFP-VASP, a fluorescent filler, mCerulean, and either mCherry or mCherry-FL-Myo10. A quantification of GFP-VASP localization (percentage) to dendritic filopodia tips from neurons expressing mCherry or mCherry-FL-Myo10 is shown. Error bars represent the s.e.m. for 30-36 cells from three separate experiments $\left({ }^{*} P<0.0001\right)$. (D) Neurons were co-transfected with GFP-VASP, mCherry-Hdl-Myo10 (mCherry-Hdl) and a fluorescent filler, mCerulean, at DIV6 and then subjected to live-cell imaging at DIV11 (top panels). Scale bar: $10 \mu \mathrm{m}$. Higher magnification images of the boxed regions are shown (lower panels). Scale bar: $5 \mu \mathrm{m}$. GFP-VASP and mCherry-Hdl-Myo10 puncta at spine heads are indicated (arrows). (E) HEK-293T cells were co-transfected with mCherry-VASP and either GFP, GFP-FL-Myo10 or GFP-Hdl-Myo10. 24 hours after transfection, cells were treated with cytochalasin D (2 $\mu$ M) for 1 hour and then lysed. GFP and GFPMyo10 isoforms were immunoprecipitated from cell lysates and subjected to immunoblot analysis. mCherry-VASP from total lysates (left panel) and immunoprecipitated complexes (upper right panel) were detected using an antibody against VASP. GFP and GFP-Myo10 isoforms from immunoprecipitated complexes were visualized with an antibody against GFP (lower right panel). (F) Quantifications of immunoprecipitated VASP from cells transfected with GFP-FLMyo10 or GFP-Hdl-Myo10 are shown. Error bars represent the s.e.m. from three separate experiments $(* P<0.02)$.

a VASP-dependent mechanism. We tested the function of VASP in FL-Myo10-mediated filopodia formation by co-transfecting DIV3-DIV4 neurons with a fluorescent filler and either GFP-FLMyo10 or GFP and VASP shRNA or NT shRNA. This shRNA against VASP has been previously shown to knock down expression of the endogenous protein by $50 \%$ in hippocampal neurons (Lin et al., 2010). Transfection of VASP shRNA into GFP-expressing neurons resulted in a decrease in filopodia density compared with that of NT-shRNA-transfected neurons (Fig. 7A,B). Moreover, knockdown of VASP in neurons expressing GFP-FL-Myo10 completely abolished the FLMyo10-promoted effect on filopodia density (Fig. 7A,B). 
A

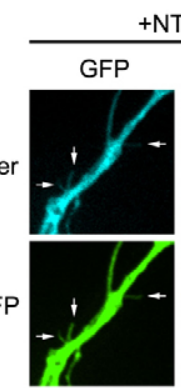

C
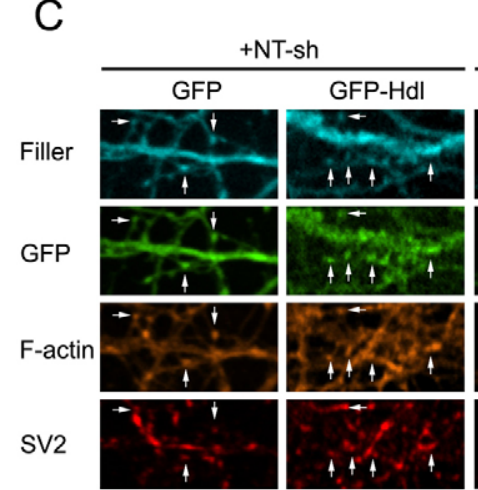

$\mathrm{F}$

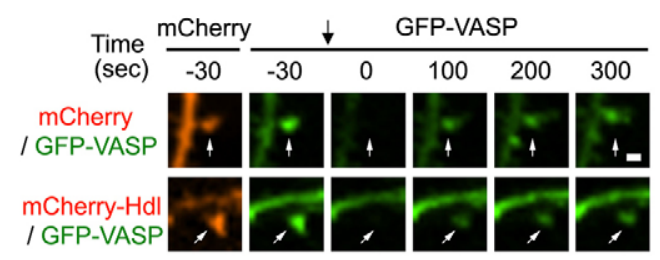

B
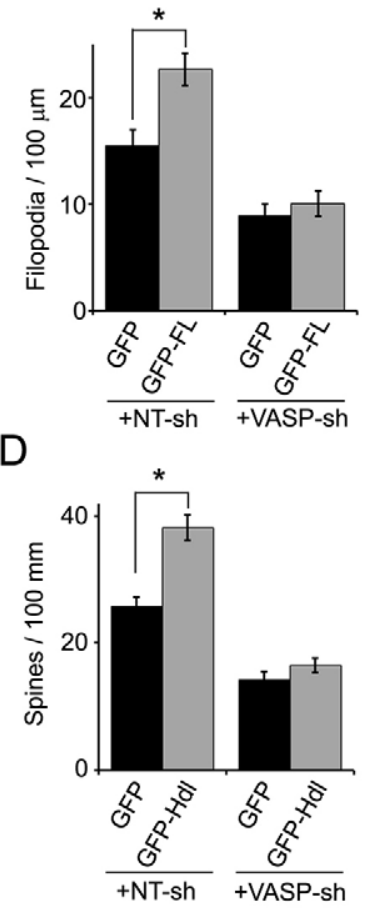

$\mathrm{E}$
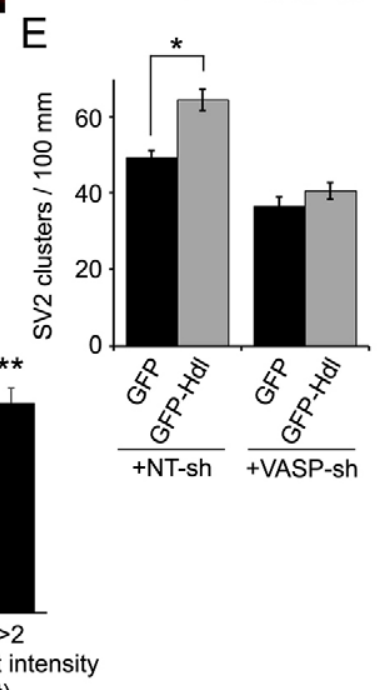

Fig. 7. FL-Myo10 and Hdl-Myo10 regulate dendritic spine and synapse development through VASP. (A) Neurons were co-transfected with a fluorescent filler, mCerulean, and either GFP or GFP-FL-Myo10 (GFP-FL) and NT shRNA (NT-sh) or VASP shRNA (VASP-sh) at DIV3-DIV4 and fixed at DIV6-DIV7.

Dendritic filopodia are indicated (arrows). Scale bar: $5 \mu \mathrm{m}$. (B) Quantifications of the density of dendritic filopodia from neurons transfected with the indicated constructs are shown. Error bars represent the s.e.m. for 44-47 dendrites from three separate experiments $\left({ }^{*} P<0.002\right)$. (C) Neurons were co-transfected with vectors fluorescent filler, mCerulean, and either GFP or GFP-Hdl-Myo10 (GFP-Hdl) and either NT shRNA or VASP shRNA at DIV3-DIV4, fixed and stained for F-actin and SV2 at DIV11-DIV12. Dendritic spines are indicated (arrows). Scale bar: $5 \mu \mathrm{m}$. (D,E) Quantifications of dendritic spine and synaptic density (SV2 clusters) from neurons transfected with the indicated constructs are shown. Error bars represent the s.e.m. for 44-47 dendrites from three separate experiments $(* P<0.0001)$. (F) Neurons were co-transfected with GFP-VASP and either mCherry-Hdl-Myo10 (mCherry-Hdl) or mCherry at DIV6, and the indicated spines (white arrows) were subjected to FRAP at DIV11. Prebleached and subsequent recovery images are shown. The photobleach point is indicated (black arrow at $t=0$ ). Scale bar: $1 \mu \mathrm{m}$. (G) Quantifications of the recovery of GFP-VASP after photobleaching in neurons expressing mCherry or mCherry-Hdl-Myo10 are shown. Error bars represent the s.e.m. from three separate experiments for 40-42 spines. (H) Neurons (DIV5-DIV6) were co-transfected with GFP-Hdl-Myo10 and a fluorescent filler, mCerulean, fixed and stained for F-actin at DIV11-DIV12. The fluorescence intensities of GFP-Hdl-Myo10 (GFP-Hdl) and F-actin were quantified in dendritic spine heads and normalized to neighboring shafts. A total of 81 spines from three separate experiments were analyzed and categorized based on their amount of GFP-Hdl-Myo10. Error bars represent the s.e.m. for $15-35$ spines from three separate experiments $(* P<0.02, * * P<0.0001)$. Asterisks indicate a statistically significant difference compared with the first category (GFP-Hdl $<1$ ). 
Since we previously demonstrated that VASP can promote the development of dendritic spines (Lin et al., 2010), this raised the question as to whether VASP was also involved in Hdl-Myo10mediated spine formation. Consistent with our previously published data (Lin et al., 2010), transfection of neurons with shRNA against VASP considerably decreased the number of dendritic spines and synapses in comparison with NT-shRNAtransfected cells (Fig. 7C-E). In addition, expression of GFPHdl-Myo10 significantly enhanced spine and synaptic density, and knockdown of VASP negated this phenotype (Fig. 7C-E), suggesting that Hdl-Myo10 promotes spine and synapse formation through VASP.

As Hdl-Myo10 increases the spine head size (Fig. 5F,G), and VASP promotes the expansion of spine heads by maintaining key synaptic proteins in spines (Lin et al., 2010), could Hdl-Myo10 promote spine head expansion by retaining VASP in spines? We coexpressed GFP-VASP along with either mCherry or mCherryHdl-Myo10 and used fluorescence recovery after photobleaching (FRAP) to examine the effect of Hdl-Myo10 on VASP retention in spines. GFP-VASP recovery was significantly slower in neurons expressing mCherry-Hdl-Myol0 than in mCherryexpressing neurons (Fig. 7F,G). Importantly, the amount of Factin in spine heads correlated positively with the expression level of GFP-Hdl-Myo10 (Fig. 7H). Thus, Hdl-Myo10 might promote spine head expansion by enhancing the retention of VASP in spines, which could increase actin assembly.

\section{Discussion}

The actin cytoskeleton governs the formation and morphological changes of dendritic spines as well as synaptic plasticity (Lin and Webb, 2009). However, the role of actin-based myosin motors in modulating these processes remains poorly understood. In this study, we show that two Myo10 isoforms have crucial, but distinct, roles in spine development. FL-Myo10 increases the number, length and dynamics of dendritic filopodia (Fig. 3), whereas Hdl-Myo10 functions directly on spines to increase spine and synapse density and spine head size (Fig. 5). Interestingly, knockdown of FL-Myo10 leads to a decrease in the density of spines and synapses (Fig. 2); however, FL-Myo10 expression did not affect spine head size (data not shown). This raises the question as to how FL-Myo10 affects spine development. One possibility is that the enhanced filopodia dynamics promoted by FL-Myo10 increases synaptic contact to facilitate spine and synapse formation. Consistent with this hypothesis, the dynamics of dendritic filopodia (extension, retraction and lateral movement) allow them to sample their surrounding environment to initiate synaptic contact with presynaptic terminals and to promote spine and synapse formation (Ethell and Pasquale, 2005; Ziv and Smith, 1996).

Intriguingly, the actin-remodeling protein VASP is involved in both FL-Myo10- and Hdl-Myo10-mediated spine development. FL-Myo10 delivers VASP to filopodia tips to promote formation of dendritic filopodia, whereas Hdl-Myo10 enhances VASP retention in spine heads to promote spine maturation. Our results demonstrating that FL-Myo10 induces dendritic filopodia formation through VASP are somewhat surprising. FL-Myo10 has been shown to promote filopodia formation in the absence of Ena/VASP proteins (Bohil et al., 2006; Dent et al., 2007). However, our data indicate that VASP is involved in FL-Myo10mediated dendritic filopodia formation in hippocampal neurons, suggesting that different underlying mechanisms for filopodia formation exist. Nonetheless, although our data strongly implicate VASP in FL-Myo10-mediated filopodia formation, we cannot exclude the possibility that other cargo might be involved as well.

Our data show that FL-Myo10 undergoes both forward and rearward movement within dendritic filopodia. This result might seem counterintuitive because FL-Myo10, a plus-end-directed motor, would be expected to move towards filopodia tips where the plus-ends are located in non-neuronal cells (Kerber et al., 2009). However, a recent study demonstrated that the polarity of actin filaments in dendritic filopodia is different from conventional non-neuronal filopodia (Korobova and Svitkina, 2010). Some actin filaments in dendritic filopodia are oriented with their plus-ends away from the tips (Korobova and Svitkina, 2010). In addition, the actin ultrastructure and composition of actin-binding proteins in dendritic filopodia are different from those in conventional filopodia, which could potentially affect actin retrograde flow and thereby FL-Myo10 rearward movement (Kerber et al., 2009; Korobova and Svitkina, 2010; Welch et al., 1997).

In this study, we also reveal a novel biological function for Hdl-Myo10 in the regulation of dendritic spine formation and maturation. As Hdl-Myo10 lacks the motor region that harbors the actin-binding site, it might not regulate actin assembly directly. Instead, Hdl-Myo10, which has multiple protein-protein interacting regions, could serve as a scaffolding protein that regulates actin dynamics indirectly through actin-binding proteins. Indeed, our data show that Hdl-Myo10 interacts with the actin-remodeling protein VASP, and expression of HdlMyo10 increases VASP retention in spines. Moreover, increasing expression of Hdl-Myo10 in neurons leads to higher amounts of F-actin in dendritic spines, which could provide a more stable platform for the recruitment of proteins to spines. This could lead to the expansion of the postsynaptic density and enhanced spine development (Lin and Webb, 2009).

Unlike FL-Myo10, Hdl-Myo10 rarely localizes to filopodia tips and does not affect the density, length and dynamics of dendritic filopodia. Hdl-Myo10, instead, regulates spine density and spine head size. Expression of GFP-Hdl-Myo10 increases the number of spines and spine head size, whereas knockdown of Hdl-Myo10 leads to a significant decrease in spine density without affecting filopodia density and length. Through the recruitment of other proteins, such as VASP, Hdl-Myo10 could support spine maturation without directly affecting filopodia formation. While this is the most likely scenario, we cannot completely rule out the possibility that Hdl-Myo10 plays some role in filopodia development as some filopodia have small amounts of Hdl-Myo10. In addition, it is possible that some spines could arise from dendritic shafts without evolving from dendritic filopodia, as has been proposed previously (Ethell and Pasquale, 2005). However, our data strongly suggest that HdlMyo10 has an important function in the formation and maturation of dendritic spines.

In summary, we show that two Myo10 isoforms play important, but distinct, roles in spine development. FL-Myo10 regulates the formation, length and dynamics of dendritic filopodia, whereas Hdl-Myo10 modulates dendritic spine formation and spine head expansion. By enhancing filopodia dynamics, FL-Myo10 expression might facilitate synaptic contact because filopodia are thought to actively search for presynaptic terminals (Ethell and Pasquale, 2005). FL-Myo10 could regulate 
this process by trafficking VASP to the filopodia tips, where it promotes actin elongation. Hdl-Myo10, which has a stronger association with VASP, increases VASP retention in spine heads to induce spine formation and spine head expansion. In this distinct fashion, FL-Myo10 and Hdl-Myo10 promote the development of dendritic spines and synapses. Thus, this study demonstrates a new function for Myo10 isoforms in dendritic spine and synapse development and a novel biological function for Hdl-Myo10 that is independent of FL-Myo10.

\section{Materials and Methods}

\section{Reagents and constructs}

Polyclonal antibodies against Myo10 were purchased from Sigma-Aldrich (\#HPA024223, St Louis, MO) and Strategic Diagnostics (\#2243.00.02, SDIX, Newark, DE). VASP monoclonal antibody (clone 43/VASP) was from BD Biosciences (Franklin Lakes, NJ). SV2 monoclonal antibodies were obtained from Developmental Studies Hybridoma Bank (University of Iowa, Iowa City, IA). PSD95 monoclonal antibody (clone 7E3-1B8) and cytochalasin D were from EMD Millipore (Billerica, MA). IRDye 800 anti-mouse was from Rockland Immunochemicals (Gilbertsville, PA). Alexa Fluor 546 phalloidin, protein A sepharose beads and sodium azide were purchased from Sigma-Aldrich (St Louis, MO). GFP polyclonal antibody, ProLong Gold antifade, Alexa Fluor 647 antimouse antibody, Alexa Fluor 488 and 555 anti-rabbit antibodies and Alexa Fluor 680 anti-rabbit antibody were obtained from Life Technologies (Carlsbad, CA). Aqua Poly/Mount was purchased from Polysciences (Warrington, PA).

GFP-FL-Myo10 (Berg and Cheney, 2002) was modified with silent mutations at G4572A and A5748C (relative to Myo10 sequence U55042) using PCR and cloned into pT $\alpha \mathrm{S} 2$ vector (Gloster et al., 1999), which was a generous gift from Freda Miller, for neuronal expression. GFP-Hdl-Myo10 (residues 644-2052) along with the following deletion constructs - GFP-Hdl-Myo10 $\triangle \mathrm{PH}(\triangle \mathrm{aa} 1168-1491)$, GFPHdl-Myo10 $\triangle$ MyTH4 ( $\triangle$ aa1543-1687), GFP-Hdl-Myo10 $\triangle$ FERM $(\triangle$ aa1761$2052)$ and GFP-FL-Myo10 $\triangle$ Motor $(\triangle 1-748)$ - were generated from the modified Myo10 cDNA by PCR and cloned into pT $\alpha$ S2. mCherry cDNA and mCerulean3 cDNA (Markwardt et al., 2011), which were kind gifts from Roger Tsien (University of California, San Diego, CA) and David Piston (Vanderbilt University, Nashville, TN), respectively, were cloned into pT $\alpha \mathrm{S} 2$ vector for expression in neurons. mCherry-FL- and mCherry-Hdl-Myo10 cDNAs, which were from the modified Myo10 sequence, were cloned into $\mathrm{pT} \alpha \mathrm{S} 2$ vector for neuronal expression. The VASP constructs have been described previously (Lin et al., 2010).

FL-Myo10 shRNA constructs were prepared using the following 19-nucleotide target sequences: shRNA\#1: 5'-AGGAAGGTGTTGACGACAT-3' and shRNA\#2: 5'-GCCTAGACTTGATCGAGAA-3'. These target sequences are located in the motor region that is unique to FL-Myol0 and absent in the Hdl isoform. An HdlMyo10 shRNA construct was generated using the target sequence: 5'GTCAGCTGCGATACTCTTG-3', which is specific to Hdl-Myo10 because this sequence is in the $5^{\prime}$-UTR (Sousa et al., 2006). The $5^{\prime}$-UTR sequence for rat was identified based on its homology to the $5^{\prime}$-UTR of mouse Hdl-Myo10. A nontargeting shRNA contained the following sequence: 5'-CAGTCGCGTTTGCGACTGG-3'. The VASP shRNA sequence 5'-TGAAAGAGGAAATAATCGA-3' has been described previously (Lin et al., 2010). For all shRNA constructs, sense and antisense oligonucleotides, containing the target sequences, were prepared and ligated into pSUPER vector, as described previously (Brummelkamp et al., 2002).

\section{Neuronal cell culture and transfection}

Rat hippocampal neurons were isolated from day-19 embryos, plated on poly-Llysine-coated $(1 \mathrm{mg} / \mathrm{ml})$ glass coverslips and maintained in culture as described previously (Goslin et al., 1998; Lin et al., 2010). Primary glia (astrocytes) were isolated from day 1-2 rat pups as described previously (Goslin et al., 1998) and maintained in culture in Minimum Essential Medium (Life technology, Carlsbad, CA) supplemented with $10 \%$ horse serum, $2 \%$ glucose and penicillin/streptomycin. HEK-293T cells were cultured in Dulbecco's Modified Eagle's Medium (Life technology, Carlsbad, CA) supplemented with $10 \%$ fetal bovine serum and penicillin-streptomycin. Neurons were transfected with a modified calcium phosphate method as described previously (Lin et al., 2010) at DIV3-DIV4 for shRNA experiments and at DIV5-DIV6 for expression experiments, unless otherwise indicated. Astrocytes and HEK-293T cells were transfected using Lipofectamine 2000 (Life technology, Carlsbad, CA) according to the manufacturer's instructions. For astrocytes, the transfection medium was replaced with fresh culture medium 4-6 hours after transfection.

\section{Immunoprecipitation}

HEK-293T cells were transfected with mCherry-VASP and either GFP, GFP-FLMyo10, or GFP-Hdl-Myo10 with Lipofectamine 2000. Twenty four hours later, cells were lysed with $40 \mathrm{mM}$ HEPES, $75 \mathrm{mM} \mathrm{KCl}, 1 \%$ TritonX-100, $2 \mathrm{mM}$
EGTA-K, $2.5 \mathrm{mM} \mathrm{MgCl} 2,2 \mathrm{mM}$ DTT, $5 \mathrm{mM}$ ATP, with protease inhibitors, $\mathrm{pH}$ 7.4. To depolymerize actin filaments, cells were treated with $2 \mu \mathrm{M}$ cytochalasin $\mathrm{D}$ for 1 hour before lysis. Cell lysates were pre-cleared by incubation for 2 hours at $4{ }^{\circ} \mathrm{C}$ with protein $\mathrm{A}$ sepharose in phosphate-buffered saline (PBS), pH 7.4 with $0.1 \%$ BSA and $0.01 \%$ sodium azide. After pre-clearing, GFP-Myo10 was immunoprecipitated from lysates by incubation with antibody to GFP $(2 \mu \mathrm{g})$ overnight at $4{ }^{\circ} \mathrm{C}$ followed by incubation with protein A sepharose for 2 hours at $4^{\circ} \mathrm{C}$. Precipitated samples were subjected to SDS-PAGE and immunoblotted for VASP and GFP. The expression levels of VASP and Myo10 in each blot were quantified using an Odyssey infrared imaging system (LI-COR, Lincoln, NE) and Odyssey application software version 3.0.29. To correct for inter-sample differences in mCherry-VASP expression levels and the amount of immunoprecipitated Myo10, the VASP band intensity in the immunoprecipitated samples was first normalized to the total amount of VASP in cell lysates and then normalized to the amount of GFP-Myo10 in the immunoprecipitation samples.

\section{Immunocytochemistry}

For most antibodies and F-actin (phalloidin) staining, neurons were fixed in 4\% paraformaldehyde in $0.12 \mathrm{M}$ sucrose in PBS for 15 minutes at $23^{\circ} \mathrm{C}$ and then permeabilized with $0.2 \%$ Triton-X-100 in PBS for 5 minutes at $23^{\circ} \mathrm{C}$. For PSD95 staining, neurons were fixed in $4 \%$ paraformaldehyde in $0.12 \mathrm{M}$ sucrose for 5 minutes at $23^{\circ} \mathrm{C}$, followed by incubation with methanol for 10 minutes at $-20^{\circ} \mathrm{C}$. To block nonspecific antibody binding, coverslips were incubated with $20 \%$ goat serum in PBS containing $0.2 \%$ Triton-X-100. Both primary and secondary antibodies were diluted in $5 \%$ goat serum in PBS with $0.2 \%$ Triton-X-100. For endogenous Myo10 staining, PBS containing 5\% BSA, $1 \%$ goat serum and $0.2 \%$ Triton-100 was used for blocking and diluting antibodies. Myo10 antibody from Strategic Diagnostics was used for endogenous Myo10 staining. Coverslips were mounted with Prolong Gold anti-fade or Aqua-Poly/Mount.

\section{Microscopy and live-cell imaging}

Neuronal imaging was performed on a Quorum WaveFX-X1 spinning disk confocal system with a Yokogawa CSU-X1 spinning disk (Yokogawa Electric Corporation, Newnan, GA) with Borealis upgrade/modifications (Guelph, Canada) or on an inverted Olympus IX71 microscope (Melville, NY). Images were acquired on the Quorum spinning disk confocal system using an EM-CCD camera (Hamamatsu, Hamamatsu City, Japan) on a Nikon Eclipse Ti microscope (Melville, NY) with MetaMorph software (Molecular Devices, Sunnyvale, CA) and a Plan Fluor $40 \times$ objective (NA 1.3) or Apo TIRF $60 \times$ objective (NA 1.49). Images for mCerulean, GFP, red fluorophores (mCherry or Alexa Fluor 546 or 555) and Alexa Fluor 647 were acquired by exciting laser lines at 405 or $441 \mathrm{~nm}$, $491 \mathrm{~nm}, 561 \mathrm{~nm}$ and $642 \mathrm{~nm}$, respectively (Semrock, Rochester, NY). Emission filters for these fluorophores were $460 / 50$ or $470 / 24,525 / 50,593 / 40$ or $620 / 60$ and 700/75, respectively (Semrock, Rochester, NY).

In some experiments, neurons were imaged on the Olympus IX71 microscope with a Retiga Exi CCD camera (QImaging, Surrey, Canada) using MetaMorph software, and an Olympus PlanApo $60 \times$ OTIRFM objective (NA 1.45). For imaging of Alexa Fluor 546, a TRITC/Cy3 cube (excitation HQ545/30, emission HQ610/75, Q570LP dichroic mirror) was used. An Endow GFP Bandpass filter cube (excitation HQ470/40, emission HQ525/50, Q495LP dichroic mirror) (Chroma, Brattleboro, VT) was used for Alexa Fluor 488.

Time-lapse live-cell imaging was performed on the Quorum WaveFX-X1 spinning disk confocal system. During imaging, cells were maintained in $10 \mathrm{mM}$ HEPES, $150 \mathrm{mM} \mathrm{NaCl}, 5 \mathrm{mM} \mathrm{KCl}, 2 \mathrm{mM} \mathrm{CaCl}_{2}$ and $30 \mathrm{mM}$ glucose, $\mathrm{pH} 7.4$ (imaging media) at $37^{\circ} \mathrm{C}$ using a temperature-controlled chamber (Live Cell Instrument, Seoul, Korea). For time-lapse imaging of filopodia dynamics, images were acquired at 3-10 second intervals. For time-lapse imaging of Myo10 and VASP trafficking, images were acquired at 5 second intervals.

\section{Fluorescence recovery after photobleaching}

FRAP was performed on the Quorum spinning disk confocal system with a Nikon Eclipse Ti microscope and an Apo TIRF $60 \times$ objective. Neurons at DIV11 were maintained in imaging media at $37^{\circ} \mathrm{C}$. Five circular regions of interest (11 pixels in diameter) in spine heads were subjected to photobleaching using a 405-nm diode laser for a 2000 millisecond pulse with $100 \%$ laser power. Following photobleaching, images were taken every 10 seconds for 5 minutes using an EMCCD camera (Hamamatsu, Hamamatsu City, Japan) and MetaMorph software. Images were taken before bleaching to provide prebleached reference images. In addition, an mCherry image was obtained to identify spines expressing mCherry and mCherry-Hdl-Myo10. To analyze FRAP, the background-subtracted fluorescence intensity of photobleached spines was corrected for fluorescence decay that resulted from image acquisition. The corrected data at each time point $(t)$ were then graphed to show recovery traces as the percentage of prebleached signal.

\section{Image analysis}

Linescan analysis was performed using MetaMorph software to assess the localization of GFP-Myo10 isoforms. The background-subtracted average 
fluorescence intensity was obtained from a 2-pixel (width $0.3 \mu \mathrm{m}$ ) line drawn from the base to the tip of protrusions. The density of dendritic filopodia and spines and synapses was quantified along primary and secondary dendrites. Specifically, dendritic filopodia were defined as protrusions without a noticeable bulbous head, whereas dendritic spines were identified as protrusions with a bulbous head that colocalized with synaptic proteins (SV2 or PSD95). To identify protrusions, neurons were either stained with phalloidin or co-transfected with F-tractin to show F-actin. F-actin is enriched at spine heads and serves as a marker for dendritic spines, but it is not enriched at filopodia tips (Allison et al., 1998; Fischer et al., 1998). Staining was considered positive only if the average fluorescence intensity was at least twofold higher than the surrounding background in dendrites. To analyze dendritic filopodia movement, the length of filopodia was manually measured from individual images as a function of time. For VASP targeting experiments, Hdl-Myo10 knockdown in spines was confirmed by immunostaining for Myo10, and VASP localization to spine heads was analyzed as described previously (Lin et al., 2010). Statistical analyses were performed using a Student's $t$-test. Data are shown as means \pm s.e.m.

\section{Acknowledgements}

We are grateful to Freda Miller, David Piston and Roger Tsien for giving us reagents. We thank $\mathrm{Lan} \mathrm{Hu}$ for technical assistance with the preparation of neuronal cultures. We thank Monica de Silva for assistance with generating cDNA constructs.

\section{Author contributions}

W.-H.L, J.T.H., and D.J.W. designed the experiments. W.-H.L. and J.T.H. performed all of the experiments and analyzed the data R.E.C. and A.N.R. provided scientific input, Myo10 constructs, and manuscript suggestions. W.-H.L. and D.J.W. wrote the paper.

\section{Funding}

This work was supported by National Institutes of Health [grant numbers GM092914, MH071674 to D.J.W.; DC03299 to R.E.C.]; and by National Center for Research Resources [grant number S10RR025524 to D.J.W.] Deposited in PMC for release after 12 months.

\section{Supplementary material available online at}

http://jcs.biologists.org/lookup/suppl/doi:10.1242/jcs.132969/-/DC1

\section{References}

Allison, D. W., Gelfand, V. I., Spector, I. and Craig, A. M. (1998). Role of actin in anchoring postsynaptic receptors in cultured hippocampal neurons: differential attachment of NMDA versus AMPA receptors. J. Neurosci. 18, 2423-2436.

Almagro, S., Durmort, C., Chervin-Pétinot, A., Heyraud, S., Dubois, M., Lambert, O., Maillefaud, C., Hewat, E., Schaal, J. P., Huber, P. et al. (2010). The motor protein myosin-X transports VE-cadherin along filopodia to allow the formation of early endothelial cell-cell contacts. Mol. Cell. Biol. 30, 1703-1717.

Berg, J. S. and Cheney, R. E. (2002). Myosin-X is an unconventional myosin that undergoes intrafilopodial motility. Nat. Cell Biol. 4, 246-250.

Berg, J. S., Derfler, B. H., Pennisi, C. M., Corey, D. P. and Cheney, R. E. (2000) Myosin-X, a novel myosin with pleckstrin homology domains, associates with regions of dynamic actin. J. Cell Sci. 113, 3439-3451.

Bohil, A. B., Robertson, B. W. and Cheney, R. E. (2006). Myosin-X is a molecular motor that functions in filopodia formation. Proc. Natl. Acad. Sci. USA 103, 12411 12416.

Brummelkamp, T. R., Bernards, R. and Agami, R. (2002). A system for stable expression of short interfering RNAs in mammalian cells. Science 296, 550-553.

Dent, E. W., Kwiatkowski, A. V., Mebane, L. M., Philippar, U., Barzik, M., Rubinson, D. A., Gupton, S., Van Veen, J. E., Furman, C., Zhang, J. et al. (2007) Filopodia are required for cortical neurite initiation. Nat. Cell Biol. 9, 1347-1359.

Ethell, I. M. and Pasquale, E. B. (2005). Molecular mechanisms of dendritic spine development and remodeling. Prog. Neurobiol. 75, 161-205.

Fiala, J. C., Spacek, J. and Harris, K. M. (2002). Dendritic spine pathology: cause or consequence of neurological disorders? Brain Res. Brain Res. Rev. 39, 29-54

Fischer, M., Kaech, S., Knutti, D. and Matus, A. (1998). Rapid actin-based plasticity in dendritic spines. Neuron 20, 847-854.

Gloster, A., El-Bizri, H., Bamji, S. X., Rogers, D. and Miller, F. D. (1999). Early induction of Talpha1 alpha-tubulin transcription in neurons of the developing nervous system. J. Comp. Neurol. 405, 45-60.

Goslin, K., Asmussen, H. and Banker, G. (1998). Rat Hippocampal Neurons in LowDensity Culture. Cambridge, MA: MIT Press.

Gray, E. G. (1959). Axo-somatic and axo-dendritic synapses of the cerebral cortex: an electron microscope study. J. Anat. 93, 420-433.

Harris, K. M. and Kater, S. B. (1994). Dendritic spines: cellular specializations imparting both stability and flexibility to synaptic function. Annu. Rev. Neurosci. 17, 341-371.
Hirano, Y., Hatano, T., Takahashi, A., Toriyama, M., Inagaki, N. and Hakoshima, T. (2011). Structural basis of cargo recognition by the myosin-X MyTH4-FERM domain. EMBO J. 30, 2734-2747.

Hodges, J. L., Newell-Litwa, K., Asmussen, H., Vicente-Manzanares, M. and Horwitz, A. R. (2011). Myosin IIb activity and phosphorylation status determines dendritic spine and post-synaptic density morphology. PLOS ONE 6, e24149.

Homma, K., Saito, J., Ikebe, R. and Ikebe, M. (2001). Motor function and regulation of myosin X. J. Biol. Chem. 276, 34348-34354.

Ju, X. D., Guo, Y., Wang, N. N., Huang, Y., Lai, M. M., Zhai, Y. H., Guo, Y. G., Zhang, J. H., Cao, R. J., Yu, H. L. et al. (2013). Both myosin-10 isoforms are required for radial neuronal migration in the developing cerebral cortex. Cereb. Cortex [Epub ahead of print] doi:10.1093/cercor/bhs407.

Kerber, M. L. and Cheney, R. E. (2011). Myosin-X: a MyTH-FERM myosin at the tips of filopodia. J. Cell Sci. 124, 3733-3741.

Kerber, M. L., Jacobs, D. T., Campagnola, L., Dunn, B. D., Yin, T., Sousa, A. D., Quintero, O. A. and Cheney, R. E. (2009). A novel form of motility in filopodia revealed by imaging myosin-X at the single-molecule level. Curr. Biol. 19, 967-973.

Korobova, F. and Svitkina, T. (2010). Molecular architecture of synaptic actin cytoskeleton in hippocampal neurons reveals a mechanism of dendritic spine morphogenesis. Mol. Biol. Cell 21, 165-176.

Kovács, M., Wang, F. and Sellers, J. R. (2005). Mechanism of action of myosin X, a membrane-associated molecular motor. J. Biol. Chem. 280, 15071-15083.

Lin, W. H. and Webb, D. J. (2009). Actin and actin-binding proteins: masters of dendritic spine formation, morphology, and function. Open Neurosci J. 3, 54-66.

Lin, Y. L., Lei, Y. T., Hong, C. J. and Hsueh, Y. P. (2007). Syndecan-2 induces filopodia and dendritic spine formation via the neurofibromin-PKA-Ena/VASP pathway. J. Cell Biol. 177, 829-841.

Lin, W. H., Nebhan, C. A., Anderson, B. R. and Webb, D. J. (2010). Vasodilator-stimulated phosphoprotein (VASP) induces actin assembly in dendritic spines to promote their development and potentiate synaptic strength. J. Biol. Chem. 285, 36010-36020.

Lisé, M. F., Wong, T. P., Trinh, A., Hines, R. M., Liu, L., Kang, R., Hines, D. J., Lu, J., Goldenring, J. R., Wang, Y. T. et al. (2006). Involvement of myosin Vb in glutamate receptor trafficking. J. Biol. Chem. 281, 3669-3678.

Lu, Q., Yu, J., Yan, J., Wei, Z. and Zhang, M. (2011). Structural basis of the myosin X $\mathrm{PH} 1(\mathrm{~N})-\mathrm{PH} 2-\mathrm{PH} 1(\mathrm{C})$ tandem as a specific and acute cellular $\mathrm{PI}(3,4,5) \mathrm{P}(3)$ sensor. Mol. Biol. Cell 22, 4268-4278.

Mallavarapu, A. and Mitchison, T. (1999). Regulated actin cytoskeleton assembly at filopodium tips controls their extension and retraction. J. Cell Biol. 146, 1097-1106.

Markwardt, M. L., Kremers, G. J., Kraft, C. A., Ray, K., Cranfill, P. J., Wilson, K. A., Day, R. N., Wachter, R. M., Davidson, M. W. and Rizzo, M. A. (2011). An improved cerulean fluorescent protein with enhanced brightness and reduced reversible photoswitching. PLOS ONE 6, e17896.

Nagy, S., Ricca, B. L., Norstrom, M. F., Courson, D. S., Brawley, C. M., Smithback, P. A. and Rock, R. S. (2008). A myosin motor that selects bundled actin for motility. Proc. Natl. Acad. Sci. USA 105, 9616-9620.

Osterweil, E., Wells, D. G. and Mooseker, M. S. (2005). A role for myosin VI in postsynaptic structure and glutamate receptor endocytosis. J. Cell Biol. 168, 329-338.

Pi, X., Ren, R., Kelley, R., Zhang, C., Moser, M., Bohil, A. B., Divito, M., Cheney, R. E. and Patterson, C. (2007). Sequential roles for myosin-X in BMP6-dependent filopodial extension, migration, and activation of BMP receptors. J. Cell Biol. 179, $1569-1582$.

Raines, A. N., Nagdas, S., Kerber, M. L. and Cheney, R. E. (2012). Headless Myo10 is a negative regulator of full-length Myo10 and inhibits axon outgrowth in cortica neurons. J. Biol. Chem. 287, 24873-24883.

Rex, C. S., Gavin, C. F., Rubio, M. D., Kramar, E. A., Chen, L. Y., Jia, Y., Huganir, R. L., Muzyczka, N., Gall, C. M., Miller, C. A. et al. (2010). Myosin IIb regulates actin dynamics during synaptic plasticity and memory formation. Neuron 67, 603-617.

Rogers, M. S. and Strehler, E. E. (2001). The tumor-sensitive calmodulin-like protein is a specific light chain of human unconventional myosin X. J. Biol. Chem. 276, 12182-12189.

Rubio, M. D., Johnson, R., Miller, C. A., Huganir, R. L. and Rumbaugh, G. (2011). Regulation of synapse structure and function by distinct myosin II motors. J. Neurosci. 31, 1448-1460

Ryu, J., Liu, L., Wong, T. P., Wu, D. C., Burette, A., Weinberg, R., Wang, Y. T. and Sheng, M. (2006). A critical role for myosin IIb in dendritic spine morphology and synaptic function. Neuron 49, 175-182.

Sousa, A. D., Berg, J. S., Robertson, B. W., Meeker, R. B. and Cheney, R. E. (2006). Myo10 in brain: developmental regulation, identification of a headless isoform and dynamics in neurons. J. Cell Sci. 119, 184-194.

Sun, Y., Sato, O., Ruhnow, F., Arsenault, M. E., Ikebe, M. and Goldman, Y. E. (2010). Single-molecule stepping and structural dynamics of myosin X. Nat. Struct. Mol. Biol. 17, 485-491

Tokuo, H. and Ikebe, M. (2004). Myosin X transports Mena/VASP to the tip of filopodia. Biochem. Biophys. Res. Commun. 319, 214-220.

Umeki, N., Jung, H. S., Sakai, T., Sato, O., Ikebe, R. and Ikebe, M. (2011) Phospholipid-dependent regulation of the motor activity of myosin X. Nat. Struct Mol. Biol. 18, 783-788.

Wagner, W., Brenowitz, S. D. and Hammer, J. A., III (2011). Myosin-Va transports the endoplasmic reticulum into the dendritic spines of Purkinje neurons. Nat. Cell Biol. 13, 40-48.

Wang, Z., Edwards, J. G., Riley, N., Provance, D. W., Jr, Karcher, R., Li, X. D., Davison, I. G., Ikebe, M., Mercer, J. A., Kauer, J. A. et al. (2008). Myosin Vb mobilizes recycling endosomes and AMPA receptors for postsynaptic plasticity. Cell 135, 535-548. 
Wei, Z., Yan, J., Lu, Q., Pan, L. and Zhang, M. (2011). Cargo recognition mechanism of myosin X revealed by the structure of its tail MyTH4-FERM tandem in complex with the DCC P3 domain. Proc. Natl. Acad. Sci. USA 108, 3572-3577.

Welch, M. D., Mallavarapu, A., Rosenblatt, J. and Mitchison, T. J. (1997). Actin dynamics in vivo. Curr. Opin. Cell Biol. 9, 54-61.

Zhang, H., Berg, J. S., Li, Z., Wang, Y., Lång, P., Sousa, A. D., Bhaskar, A., Cheney, R. E. and Strömblad, S. (2004). Myosin-X provides a motor-based link between integrins and the cytoskeleton. Nat. Cell Biol. 6, 523-531.
Zhu, X. J., Wang, C. Z., Dai, P. G., Xie, Y., Song, N. N., Liu, Y., Du, Q. S., Mei, L., Ding, Y. Q. and Xiong, W. C. (2007). Myosin X regulates netrin receptors and functions in axonal path-finding. Nat. Cell Biol. 9, 184-192.

Zito, K., Knott, G., Shepherd, G. M., Shenolikar, S. and Svoboda, K. (2004). Induction of spine growth and synapse formation by regulation of the spine actin cytoskeleton. Neuron 44, 321-334.

Ziv, N. E. and Smith, S. J. (1996). Evidence for a role of dendritic filopodia in synaptogenesis and spine formation. Neuron 17, 91-102. 